\title{
MEDICIÓN Y ESTIMACIÓN DE LOS ALBAÑILES, UN APORTE A LA EDUCACIÓN MATEMÁTICA
}

\section{MEASUREMENT AND ESTIMATION OF BRICK LAYERS, A CONTRIBUTION TO MATHEMATICS EDUCATION}

\author{
Miller Freddy Rey Muñoz¹, Armando Aroca Araújo²
}

\begin{abstract}
${ }^{1}$ Licenciado en Educación Básica con énfasis en Matemáticas. Profesor Colegio Campestre Anglo Hispano. Estudiante de $5^{\circ}$ semestre de Recreación en la Universidad del Valle. millerfreddy@yahoo.es ${ }^{2}$ Licenciado en Matemáticas y Física. Magíster en Educación Matemática. Profesor TC - Universidad Santiago de Cali. aaroca@usc.edu.co, aroca@etnomatematica.org
\end{abstract}

Rev. U.D.C.A Act. \& Div. Cient. 14(1): 137 - 147, 2011

\section{RESUMEN}

El presente artículo es el resultado de una investigación, cuya actividad de campo se desarrolló alrededor de seis meses, en el Distrito de Agua Blanca de Cali y en el barrio de las Américas de Palmira, Valle del Cauca, Colombia. El propósito fue determinar cómo eran algunos procesos de medición y de estimación en los albañiles. Se presentan algunas reflexiones sobre los aportes de este grupo de trabajadores a la Educación Matemática. Doce albañiles, con bajo grado de escolaridad y no coordinados por Ingenieros, colaboraron con la información. Las edificaciones que se visitaron fueron construcciones para vivienda (construcción de dos pisos o un segundo piso) y la construcción de una iglesia. Para analizar dichos procesos cognitivos, se hicieron pruebas controladas, se experimentaron situaciones reales en el oficio y conversaciones reales entre Oficiales y Ayudantes $y$, se estudiaron algunas herramientas artesanales, propias del ejercicio de la albañilería, como la flejadora, la grifa, la cimbra y la manguera de nivel. Solo se presenta una pequeña parte de ese ambiente de construcción etnomatemática, importante para el desarrollo arquitectónico de un pueblo, fecundo de discursos lógicos y gestuales y herramientas dispersas por doquier.

Palabras clave: Etnomatemática, medición, estimación, albañiles.

\section{SUMMARY}

This article is the result of a research activity realized during six months at the district of Agua Blanca (Cali) and in the suburb Americas (Palmira), Valle del Cauca, Colombia. The purpose was to determine some mason's measurements and estimation processes. Reflections about the contributions of this working group to the Mathematical Education are presented. Twelve masons, with low schooling levels and not coordinated by engineers, helped with information. The constructions visited were houses (two-story buildings or the structure of a second floor) and a church. To analyze these cognitive processes controlled trials were executed; real situations in the office and actual conversations between officers and assistants were witnessed, and some proper masonry craft tools such as the strap, the faucet, the form and plummet were studied. Only a small part of building environment related to ethnomatemathics, important for the architectural development of a, fertile of logical speeches and gestures and tools scattered everywhere, nation.

Key words: Ethnomathematics, measurement, assessment, brick layers.

\section{INTRODUCCIÓN}

La albañilería es una práctica social que tiene diversos matices en el territorio colombiano, es decir, sus técnicas y sus materiales pueden variar según la región. Es una actividad muy poco documentada, desde el análisis sistemático en sus prácticas y saberes y sus posibilidades en la educación matemática; sin embargo, en Brasil, se pueden encontrar algunas investigaciones que analizan estas perspectivas, como las de Delfino da Silva (2007), Glavam (2003), Truzzi (2006) y Pardal (2008).

En la albañilería, se presenta una jerarquía, por lo menos en el Valle del Cauca, definida descendentemente así: Maestro 
de Obra: "No se unta", coordina toda la construcción, entrega materiales; Oficial: "Sabe de todo, por ejemplo, arma cajones de las vigas y columnas y así..."; Ayudante Práctico: "Sabe armar castillos y así..." y Ayudante: "Ese es el que mezcla, carga ladrillos, bultos y eso...". Iniciarse en la albañilería implica un aprendizaje por medio de una tradición oral y empírica o de la copia de modelos ya construidos y culturalmente valorizados, sobre los cuales son introducidos variantes, sin duda originales (Knijnik, 2006).

La albañilería data desde tiempos remotos; los primeros ladrillos o bloques fueron usados en Babilonia y en el antiguo Egipto, esto significa que el aprendizaje y la construcción de nuevos significados y heurísticas que, por lo general, dependen de las condiciones geográficas o de las materias primas que el medio provea, han tenido un tiempo suficiente para ser perfeccionados, como las estimaciones al ojo, las medidas con la cuarta o dedos o la nivelación a partir de una "burbuja" de agua, como sucede con la Manguera de Nivel, la suavización de superficies inclinadas o planas con la Llana Metálica o con la Llana de Madera o Platacho, con Codales o el doblaje angular de varillas de hierro, con la Flejadora o la división de superficies planas verticales u horizontales, con la Cimbra (Figura 1).

Dar una definición de albañilería podría ser fácil, pero facilitarla sobre la etnomatemática de este grupo laboral es complejo; para el caso, se puede analizar el diálogo entre Monteiro, Orey y Do Carmo, en Machado et al. (2004), donde se presentan tres enfoques diversos sobre la etnomatemática. Sobre la pluralidad en las significaciones del programa de etnomatemática, Lunkes (2004) concluye que, así como, se debe tener una noción de qué es cultura, la etnomamatemática merece un mismo tratamiento conceptual. Para tener, entonces, una noción sobre las etnomatemáticas, vale la pena tener en cuenta a D'Ambrosio, en Blanco (2008). En ella, D'Ambrosio manifiesta que la definición de etnomatemática es compleja y que la definición que él tiene es de carácter etimológico, "como tres raíces, una de ellas es etno y por etno yo comprendo los diversos ambientes social, cultural, natural, la naturaleza, todo eso. Después hay otra raíz, que es una raíz griega que llama mathema y el griego mathema quiere decir explicar, entender, enseñar, manejarse; y un tercer componente es thica que yo introduzco ligado a la raíz griega tecni que es artes, técnicas...". Otro referente sobre el particular es Bishop (1999, 2005), donde se encuentran las seis prácticas, que él determina como universales y que generan pensamiento matemático: contar, medir, jugar, diseñar, localizar y explicar. En este mismo sentido existe una fundamentación sobre dicho campo, en Machado et al. (2004) y, tal vez, el más reciente análisis sobre las etnomatemáticas, François $\mathcal{E}$ Kerkhove (2010), muestra un panorama alentador. En la actividad de la albañilería, se encuentra con significativa expresión las actividades de diseñar y medir, procesos muy documentados, pero no tan contextualizados en la actividad escolar. Según Bishop (1999), medir es importante para el desarrollo de ideas matemáticas y se ocupa de comparar, de ordenar y de cuantificar cualidades que tienen valor e importancia. La actividad de medir, incluyendo el proceso de estimación, son actividades culturales de la humanidad que se practican de manera implícita y explícita en diversas tareas cotidianas. La importancia de esto radica que existen actividades reales, a la vuelta de la esquina de los colegios y que ellas, pueden ser llevadas al currículo matemático para el desarrollo del pensamiento métrico, dejando así de lado algunos ejemplos estériles, que no satisfacen las perspectivas o están ligados con el background cultural de los estudiantes (Skovsmose, 2004).

Los lineamientos curriculares del Ministerio de Educación Nacional (MEN) (1998) mencionan que, en la medida, los énfasis están en comprender los atributos medibles (longitud, área, capacidad, peso) y su carácter de invarianza, dar significado al patrón y a la unidad de medida y a los procesos mismos de medición; desarrollar el sentido de la medida (que implica la estimación) y las destrezas para medir, involucrar, significativamente, aspectos geométricos, como la semejanza en mediciones indirectas y los aspectos aritméticos, fundamentalmente, en lo relacionado con la ampliación del concepto de número. Con respecto a la noción de estimación, se puede considerar lo expuesto por Bright (1976), citado por el MEN (1998), quien define la estimación de magnitudes como el proceso de llegar a una medida sin la ayuda de instrumentos. Para el caso de los albañiles existen estimaciones "al ojo", el uso de instrumentos no graduados representados por partes del cuerpo, como la mano o el paso. Por su parte, Posada (2007) argumenta que el proceso de estimar es de vital importancia, pues permite acceder a complejas técnicas de medición. La estimación, entonces, como proceso de aprendizaje de otras nociones, juega un papel importante en la escuela, donde una de sus aplicaciones más importantes es la de usarse después de haber comprendido el sistema métrico decimal, debido a que es indispensable, dar medidas aproximadas sin utilizar instrumentos (Chamorro $\mathcal{E}$ Belmonte, 1994); no obstante, esta afirmación tendría sus reservas con aquellos grupos que no son escolarizados, como indígenas tradicionales o aquellos que no usan el sistema métrico, como comunidades de pescadores. En síntesis, la estimación es una actividad social y cultural practicada por todas las personas.

\section{MATERIALES Y MÉTODOS}

Se analizó la aplicación del pensamiento métrico de doce 

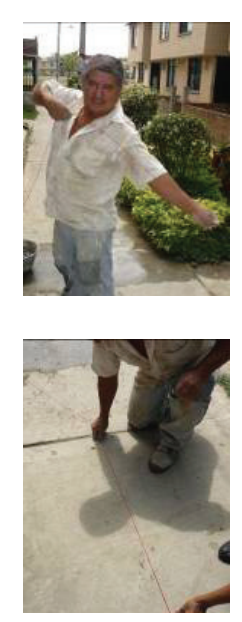

A7 muestra una cuerda y su distancia que sirve como patrón de medida, según él es un metro.

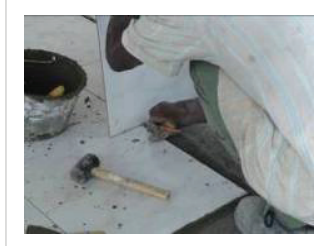

A6 mide comparando una parte de una baldosa, marca y luego corta. Enchape de un piso.

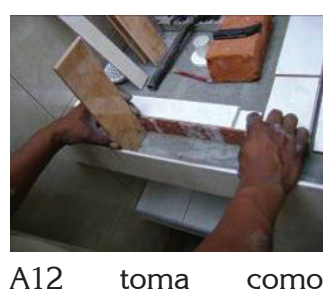

referencia el ancho de de un trozo de una baldosa para armar el borde de un lavadero.

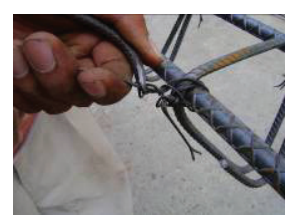

A11 hace amarres, usando pinzas artesanales, de flejes triangulares a una viga de amarre.

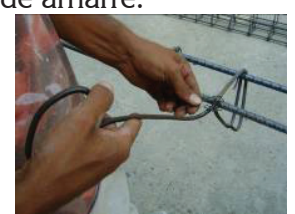

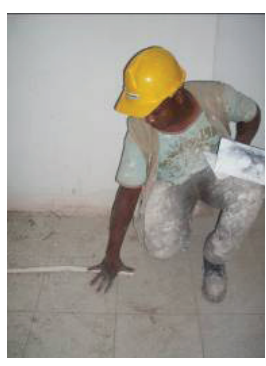

A4 mide usando su cuarta.
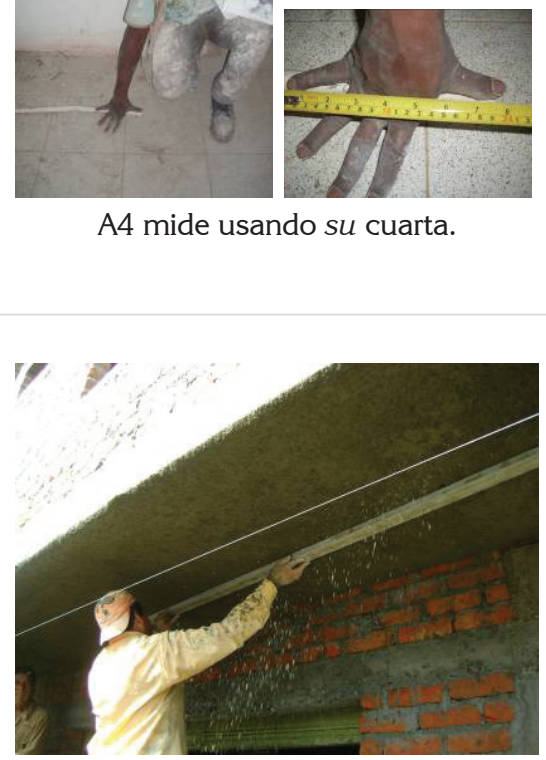

A10 repella con un Codal la superficie inferior de una placa. Lo deja de usar cuando no vea "lucecitas" entre la placa y el Codal.

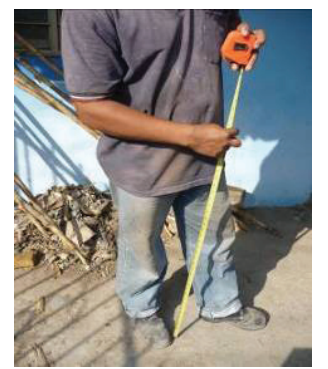

A5 toma como referencia la altura de su ombligo.

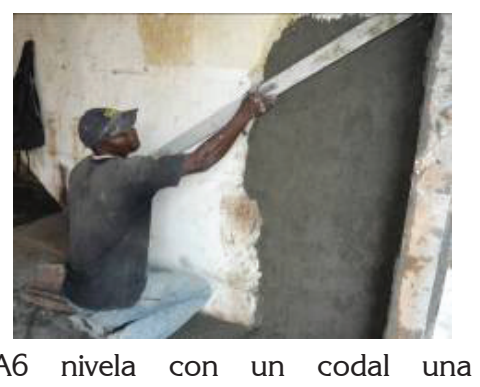
reconstrucción de una vieja pared.
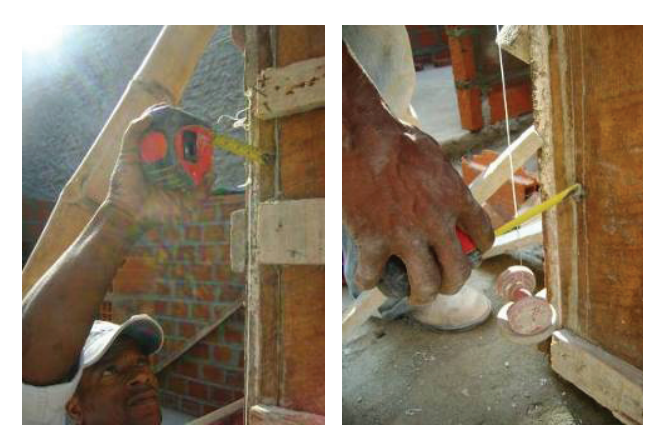

A8 hace dos mediciones, arriba y abajo, desde la pared hasta la cuerda de la Plomada, para constatar si son las mismas.

Figura 1: Algunas formas de medición o estimación. 
albañiles, de bajo grado de escolaridad, en el desempeño de sus labores en algunos barrios de la ciudad de Cali, en el Distrito de Agua Blanca, particularmente, en los barrios Mojica y Comuneros y, en la ciudad de Palmira, en el barrio las Américas, durante periodos de 2009B y 2010A. La tabla 1 , muestra más detalles sobre el particular.

Los nombres de los albañiles fueron cambiados y se nombraron A1, haciendo referencia al primer albañil y, así sucesivamente. Se tuvo en cuenta a Goetz $\mathcal{E}$ LeCompte (1998), y se empleó la metodología que admitiera la utilización de una pluralidad de instrumentos (entrevistas, grabaciones, fotos digitales, apuntes de campo), permitiendo así la recolección de datos y posibilitando la construcción y la reconstrucción de hipótesis, además de precisar, modificar y profundizar en el objeto de investigación. Por cada albañil entrevistado, se hacían las correcciones para abordar el próximo albañil, pues el Oficial temía en ciertas ocasiones por la llegada del Maestro de Obra y por el retraso a la construcción que podía implicar dar la entrevista; los Ayudantes, por su parte, en ciertas ocasiones, delegaban la "verdad" al Oficial. El trabajo fue de carácter etnográfico; se usaron entrevistas semi estructuradas, la observación fue participante, salvo en aquellos casos donde no fue posible; algo similar a lo establecido por Pardal (2008).

La metodología concuerda con lo mencionado por Ubiratan D'Ambrosio, en la entrevista concedida a Blanco (2008), donde afirmó que un método de trabajo en etnomatemática es la observación de prácticas de grupos naturales diferenciados; intenta ver qué hacen, lo qué hacen, que ellos hagan una narrativa de sus prácticas y, luego, un análisis del discurso. Para obtener elementos de análisis sobre la estimación y la medición de longitudes, se les interrogó y se les observó sobre situaciones acordes a su propio contexto. Se analizaron aquellos contextos donde no se usaba herramienta de medida, como la cinta métrica; un grupo de albañiles fue interrogado sobre la funcionalidad y la creación

Tabla 1: Algunos datos de los albañiles entrevistados.

\begin{tabular}{|c|c|}
\hline ALBAÑIL & CARACTERÍSTICAS \\
\hline $\begin{array}{l}\text { Albañil No. } 1 \text { (A1): } \\
\quad \text { Omar }\end{array}$ & $\begin{array}{l}\text { Edad: } 53 \text { años. Experiencia: } 25 \text { años. Escolaridad: Quinto de primaria. } \\
\text { Dirección de la obra: Barrio Mojica II, Cali. Trabajo realizado: Enchape de templo cristiano. Fecha y tiempo de } \\
\text { entrevista: } 17 / 07 / 09 \text {. 1h 40 }\end{array}$ \\
\hline $\begin{array}{l}\text { Albañil No. } 2 \text { (A2): } \\
\text { Fernando Ruíz }\end{array}$ & $\begin{array}{l}\text { Edad: } 48 \text { años. Experiencia: } 25 \text { años. Escolaridad: Quinto de primaria } \\
\text { Dirección de la obra: Barrio Mojica II, Cali. Trabajo realizado: Enchape de templo cristiano. Fecha y tiempo de } \\
\text { la entrevista: } 17 / 07 / 0 \text {. } 1 \mathrm{~h} 40^{\prime}\end{array}$ \\
\hline $\begin{array}{l}\text { Albañil No. } 3 \text { (A3): } \\
\text { José }\end{array}$ & $\begin{array}{l}\text { Edad: } 50 \text { años. Experiencia: } 15 \text { a } 18 \text { años. Escolaridad: Quinto de primaria. Dirección de la obra: Barrio El } \\
\text { Morichal, Cali. Trabajo realizado: Construcción de antejardín. Fecha y tiempo de la entrevista: 31/07/09. 1h 30 w }\end{array}$ \\
\hline $\begin{array}{l}\text { Albañil No. } 4 \text { (A4): } \\
\qquad \text { Mateo }\end{array}$ & $\begin{array}{l}\text { Edad: } 33 \text { años. Experiencia: } 15 \text { años. Escolaridad: Quinto de primaria } \\
\text { Dirección de la obra: Barrio Mojica II, Cali. Trabajo realizado: Obra blanca de una casa. Fecha y tiempo de la } \\
\text { entrevista: 14/08/09. 1h 10 }\end{array}$ \\
\hline $\begin{array}{l}\text { Albañil No.5 (A5): } \\
\text { Jesús }\end{array}$ & $\begin{array}{l}\text { Edad: } 35 \text { años. Experiencia: Más de } 15 \text { años. Escolaridad: Cuarto de primaria. Dirección de la obra: Barrio Las } \\
\text { Granjas, Cali. Trabajo realizado: remodelación de un techo. Fecha y tiempo de la entrevista: 24/08/09. 1h 20'. }\end{array}$ \\
\hline $\begin{array}{l}\text { Albañil No.6 (A6): } \\
\quad \text { Pedro }\end{array}$ & $\begin{array}{l}\text { Edad: } 42 \text { años. Experiencia: } 25 \text { años. Escolaridad: cuarto de primaria. Dirección de la obra: Barrio El Guabal, } \\
\text { Cali. Trabajo realizado: Reconstrucción de una casa. Fecha y tiempo de la entrevista: 01/09/09. 1h 30' }\end{array}$ \\
\hline $\begin{array}{l}\text { Albañil No.7 (A7): } \\
\text { Miguel }\end{array}$ & $\begin{array}{l}\text { Edad: } 48 \text { años. Experiencia: } 25 \text { años. Escolaridad: Sexto. Dirección de la obra: Barrio El Jardín, Cali. Trabajo } \\
\text { realizado: Remodelación de un segundo piso. Fecha y tiempo de la entrevista: 07/09/09. 1h 20’ }\end{array}$ \\
\hline $\begin{array}{l}\text { Albañil No.8 (A8): } \\
\quad \text { Luis }\end{array}$ & $\begin{array}{l}\text { Edad: } 56 \text { años. Experiencia: } 20 \text { años. Escolaridad: Desconocida. Rango: Oficial. Dirección de la obra: Barrio las } \\
\text { Américas, Palmira. Trabajo realizado: Construcción de una casa de dos pisos. Fechas y tiempos de entrevistas: } \\
03,05 / 02 / 10.1 \mathrm{~h}, 1 \mathrm{~h} 20^{\prime} \text { (respectivamente). }\end{array}$ \\
\hline $\begin{array}{l}\text { Albañil No.9 (A9): } \\
\quad \text { Sebastián }\end{array}$ & $\begin{array}{l}\text { Experiencia: } 25 \text { años. Escolaridad: Quinto de primaria. Rango: Oficial. Dirección de la obra: Barrio las Américas, } \\
\text { Palmira. Trabajo realizado: Construcción de un segundo piso de una casa. Fecha y tiempo de entrevista: } \\
\text { 05/02/10. } 30 \text { (explicación del uso de la Cimbra). }\end{array}$ \\
\hline $\begin{array}{l}\text { Albañil No. } 10 \text { (A10): } \\
\text { El señor Martín }\end{array}$ & $\begin{array}{l}\text { Edad: } 45 \text { años. Tiempo de experiencia: } 20 \text { años. Escolaridad: Sin estudio. Rango: Oficial. Dirección de la obra: } \\
\text { Barrio las Américas, Palmira. Trabajo realizado: Construcción de una casa de dos pisos y esquinera. Fecha y } \\
\text { tiempo de entrevista: 03/02/10. 20’ (explicación del uso de un codal) }\end{array}$ \\
\hline $\begin{array}{l}\text { Albañil No.11 (A11): } \\
\text { Dailer }\end{array}$ & $\begin{array}{l}\text { Edad: } 32 \text { años. Tiempo de experiencia: } 6 \text { años. Escolaridad: Noveno. Rango: Ayudante práctico. Dirección de } \\
\text { la obra: Barrio las Américas, Palmira. Trabajo realizado: Construcción de una casa de dos pisos y esquinera. } \\
\text { Fechas y tiempos de entrevista: } 03,05 / 02 / 10.1 \mathrm{~h} 20^{\prime}\end{array}$ \\
\hline $\begin{array}{l}\text { Albañil No. } 12 \text { (A12): } \\
\quad \text { Armando }\end{array}$ & $\begin{array}{l}\text { Edad: } 60 \text { años. Tiempo de experiencia: } 32 \text { años. Escolaridad: Primaria. Rango: Oficial. Dirección de la obra: } \\
\text { Barrio las Américas, Palmira. Trabajo realizado: Construcción de una casa de dos pisos y esquinera. Fechas y } \\
\text { tiempos de entrevista: 05,08/02/10. 1h 30’ (explicación de enchape). }\end{array}$ \\
\hline
\end{tabular}


de algunas herramientas artesanales de medición. El análisis de las conversaciones entre los albañiles fue un elemento que aportó, de forma significativa, para el alcance de los objetivos.

\section{RESULTADOS Y DISCUSIÓN}

Sobre algunas herramientas de los albañiles: La flejadora, la grifa, la cimbra y la manguera de nivel: Los albañiles emplean herramientas graduadas y no graduadas: llaves de amarre, la flejadora, la manguera de nivel, el nivel de mano, la cinta métrica, la cimbra, la escuadra y otras, que son medios de estimación, como el nylon, el martillo, la carretilla, la pala, la tenaza de mano, el palustre, entre otros. No hay lugar a dudas que la herramienta de medida graduada más explotada por ellos es la cinta métrica de 5 u $8 \mathrm{~m}$ de longitud. Pero hay cuatro de ellas que llamaron mucho nuestra atención, como la flejadora, la grifa, la cimbra y la manguera de nivel o el nivel de manguera; tal vez sean estos los instrumentos más artesanales e ingeniosos del oficio de la albañilería. Todos son objetos que sirven para medir, pero la estimación se involucra a cada momento. Cada uno de ellos, se aplica de forma diferente y en situaciones diversas. Se describirá a continuación, tanto su forma de creación como su empleo y, en lo posible, se harán algunas conclusiones sobre la lógica empleada en su utilización. Son estos pues instrumentos que escapan de la estandarización de instrumentos geométricos que propone el sistema escolar y de un "lenguaje universal", como lo plantea Truzzi (2006).

La flejadora y la grifa: La flejadora, se elabora con cuatro láminas gruesas de hierro, llamadas platinas, dos planas y dos angulares rectas. Las planas son separadas paralelamente a $50 \mathrm{~cm}$ (mediciones no estándares) y las platinas angulares se colocan sobre ellas perpendicularmente y se sueldan. A las planas, se les hace un orificio en cada extremo, para ser clavadas temporalmente a una tabla gruesa. Al soldarse, la separación de las platinas superiores forman un canal no muy ancho, donde se meten las varillas para ser dobladas con el tubo de flejar. Previamente, a una de las platinas angulares se le practica unos carcaones o muescas, que sirven para hacer los doblajes estimados, que producen simetría. Para hacer los carcaones, se miden y se hacen las marcas con lápiz rojo y luego son cortadas con segueta. Cada carcaón, se coloca a distancias de 10, 15, 20, 25, 30 y 35cm. Estas distancias, entonces, serán heredadas por las longitudes de los flejes, que se han de usar para columnas o vigas
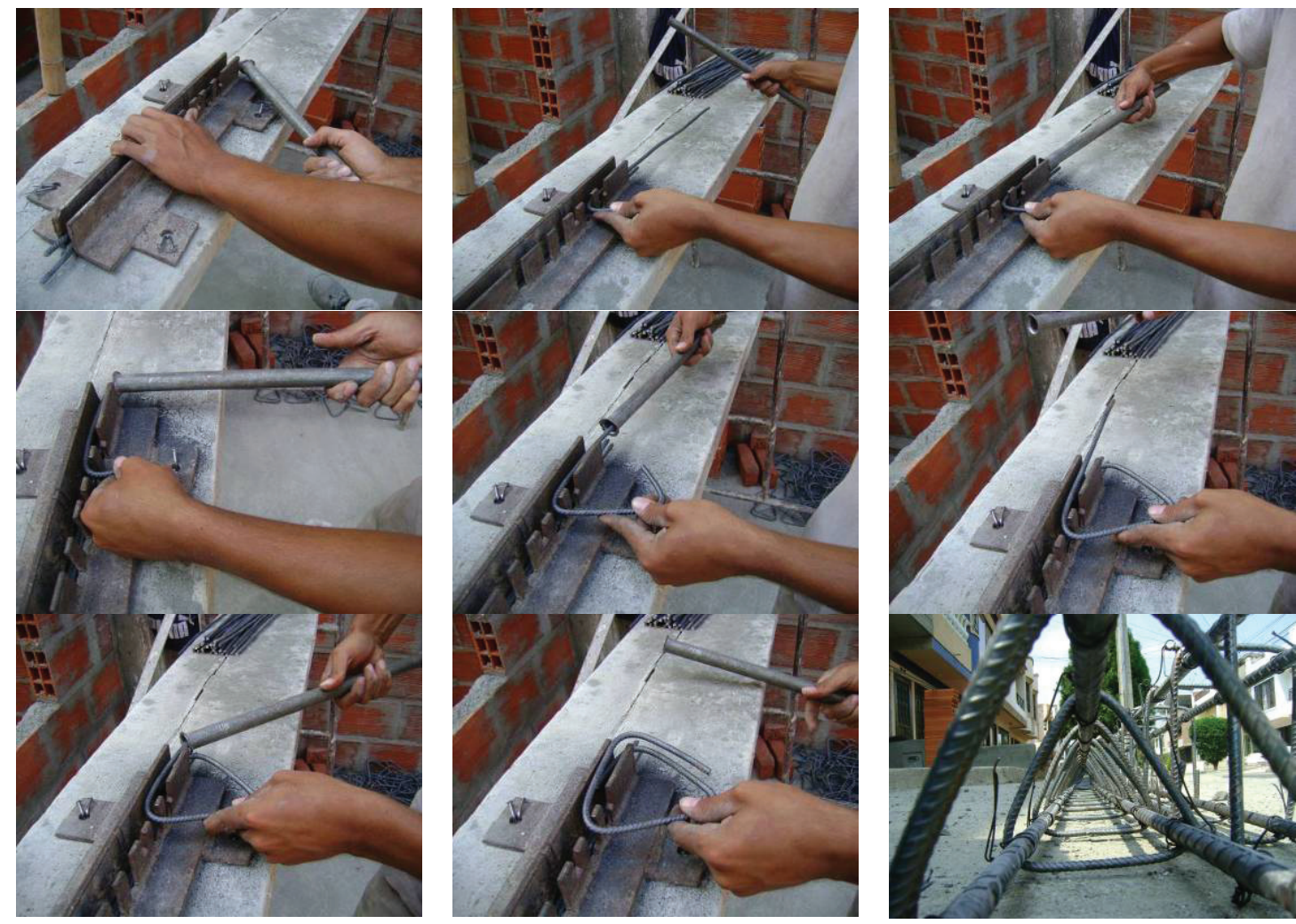

Figura 2. Uso de la flejadora para hacer flejes o estribos triangulares. Trabajo de un Ayudante práctico A11. 
de diversos grosores. Con una flejadora, se pueden hacer flejes o estribos (triángulos, eses (nervios) y cuadrados), al doblar segmentos de $50 \mathrm{~cm}$ de varillas de $1 / 4$ o $3 / 8$. Como la varilla de $1 / 2$ (media) no entra en la flejadora, pues se debe garantizar que las varillas de $1 / 4$ y $3 / 8$ no queden flojas o no tengan "juego" para optimizar su doblaje, entonces, se dobla con una grifa. El doblaje inicial, a veces, se hace hasta con tres varillas, que permite acelerar el proceso. La grifa, se hace con un metro de una varilla de una pulgada; en sus puntas hay una $U$, que si las uniéramos formarían un ángulo recto; esta gruesa varilla es doblada en un taller metalúrgico. Tanto la flejadora como la grifa tienen sus propios tubos de flejar. La figura 2 muestra el uso de la flejadora.

La elaboración de un fleje, de cualquier forma, implica un proceso que mezcla, tanto la medición como la de estimación, pues las longitudes de los lados de flejes triangulares o rectangulares son estándares y los da la flejadora, pero el ángulo de doblaje depende del cálculo referencial a un punto cualquiera de la base de la flejadora y esto se hace por estimación; eso se ve, por ejemplo, en los flejes en ese.

La cimbra: Es un instrumento geométrico semi ejecutable, que sirve para trazar rectas de 3 hasta de $20 \mathrm{~m}$, que tiene una extraordinaria diferencia de una regla o codal y es que "ella misma", a partir de una tensión a la cuerda que incluye, marca la línea sobre paredes o pisos. En una clase de geometría, el compás es el único instrumento semi ejecutable, pero la diferencia radica en que éste solo permite trazos curvos y, la cimbra, rectos. Sin duda alguna, la diferencia de los dos instrumentos radica en el tipo de superficies de trabajo, pues el compás, generalmente, se emplea sobre hojas, mientras que la cimbra sobre paredes o pisos. Esto motiva para preguntar: ċacaso no es posible trasladar la clase de geometría a otras superficies u otros lugares? La figura 3 muestra el proceso de cómo marcar una línea roja sobre una superficie cualquiera, usando la cimbra.

El papel que juega la cimbra en una construcción es vital, pues está en juego, si la cimbrada es en la pared, las construcciones a nivel de las placas de concreto que soportarán los pisos sucesivos. Un desnivel en las placas puede, incluso, repercutir en la caída parcial de la misma obra. Cuando la cimbra es empleada para cimbrar pisos, el propósito es para hacer el trazo de los pasillos internos de una vivienda. Es así, como se consigue que paredes o entradas a cuartos, cocinas, salas de estudio, queden sobre una línea. La elaboración de la cimbra, cuando es de carácter artesanal, es muy simple como ingeniosa (Figura 3).

La manguera de nivel: Esta se usa, básicamente, para levantar una pared. Ella es un instrumento muy sencillo: una manguera delgada y transparente llena de agua en casi su totalidad. La frase "viejo Jairo dame un nivel", se puede explicar con un caso particular: Hay tres columnas en la parte frontal de una casa, que apenas se construye, entonces, no hay pared, por tanto, se recurre a la manguera de nivel para trasladar una medida de la primera a la segunda columna.

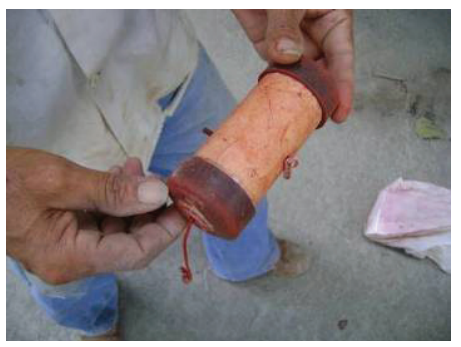

Cimbra

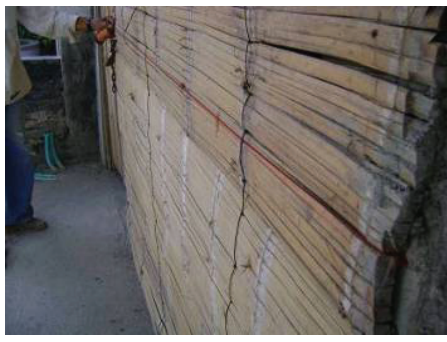

Estirando la cuerda sobre una superficie

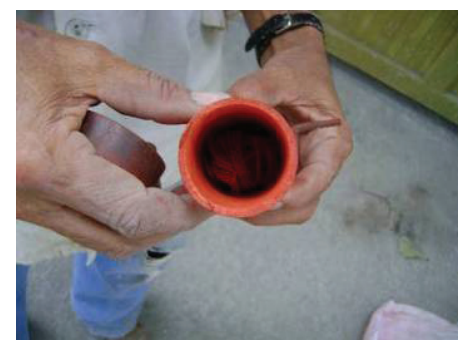

Interior

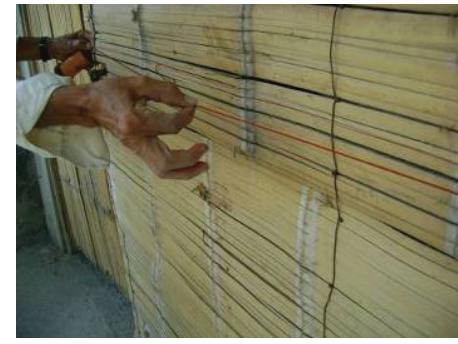

Tensionando un arco con la cuerda

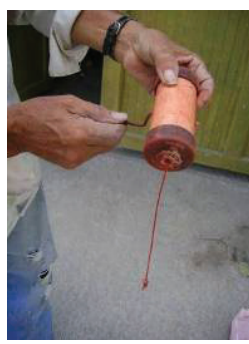

Desenrolle

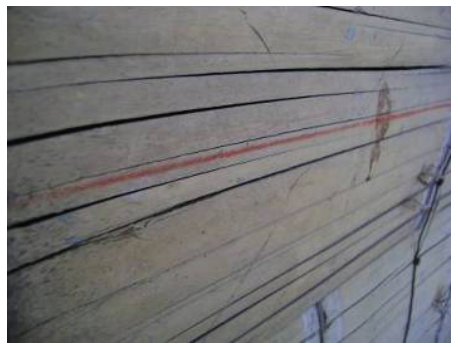

Marca de la cuerda al golpear la superficie

Figura 3. Funcionalidad de la cimbra. 
Inicialmente, se hace una marca que servirá de referencia; luego, una persona hace coincidir la "burbuja" (que no existe, pero es uno de los extremos del agua que está al interior de la manguera) con dicha marca, no sin antes de que esta burbuja se haya movido varias veces de arriba a abajo y viceversa; entonces, el Ayudante también sube y baja la manguera de nivel hasta que coincida, tanto la burbuja como la marca de referencia; cuando esto pasa, el Ayudante le hace una señal al Oficial, quien hace una segunda marca en la segunda columna y, justo allí, queda una marca que está a nivel de la primera. Este proceso puede durar alrededor de 40 segundos. Cuando el Oficial hace la marca toma el nivel de mano y traza, sobre dicha marca, una línea sobre la columna. La figura 4 muestra el proceso de transferir una medida de una columna a otra.

Medir con la manguera de nivel es una forma más de la versatilidad que tienen los albañiles para tomar y trasladar medidas. También, es posible transferir una medida de esta forma de una columna a un codal, el cual, serviría de esquinero para levantar una pared, donde no hay una columna. Después de esto, la plomada es usada para dar plomo a la pared, es decir, rectitud. Luego, aparece el nylon para ir levantando cada hilera de ladrillos (toletes) o farol (un ladrillo de altura de $20 \mathrm{~cm}$ ); las marcas que se hacen en el codal estarán, entonces, separadas por $22 \mathrm{~cm}$ : $20 \mathrm{~cm}$ que ocupa el farol y $2 \mathrm{~cm}$ de mezcla. Toda esta creatividad y diversas formas de medir para levantar una pared, más el lenguaje propio de la albañilería, no deja de sorprender. Al entrar en una construcción, lo que se puede concluir es que ese es uno de los espacios por excelencia de la expresión de los lenguajes matemático y geométrico del saber local, de un grupo socio-culturalmente diferenciado; es un ambiente único de lógica y de abstracción. Transferir una medida por manguera de nivel es un aporte más a la educación matemática, pues para que este proceso sea posible, se involucran, como mínimo, dos personas. La medida se construye de manera grupal.

Se encontraron tres patrones o características similares aplicadas como métodos de estimación de longitudes extensas; estos métodos son: "Al ojo", que se basa, esencialmente, en la observación de la longitud que se desea estimar, después de mirar y de calcular la posible medida; se hace una aproximación y se da una respuesta. Cabe anotar que el éxito de este tipo de estimación, se centra en la experiencia de los albañiles. Se privilegia mucho esta forma de estimación cuando el tiempo apremia o hay que hacer algún cálculo contable. "El paso largo", donde el uso de partes del cuerpo, para hacer estimaciones, juega un papel importante. Este tipo de método consiste en contar los pasos que hay de un punto a otro dentro de un espacio determinado, teniendo presente que no son pasos normales, sino un poco más alargados al momento de darlos, donde cada uno de éstos mide, según todos los albañiles, un metro, independientemente de la altura de ellos.

Los puntos o lugares de referencia son métodos o procesos de estimación, que se fundamenta en tener puntos o lugares que sirvan como referencia para estimar la posible medida de una longitud, aclarando que, en algunos casos, se debe conocer, con antelación, la medida de los lugares, la cual, debe ser de una menor medida, de tal forma que se le pueda calcular con mayor facilidad su longitud y ser utilizada como de referencia, para estimar una longitud más extensa.

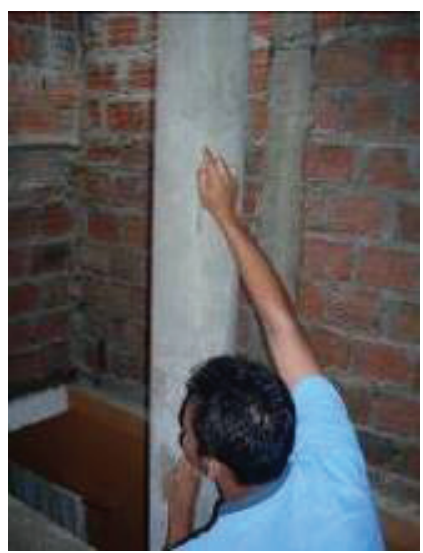

Primera persona en el sitio inicial.

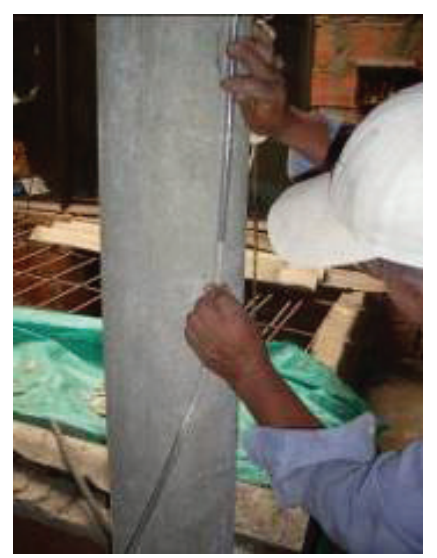

Segunda persona en el sitio donde se desea transferir la medida.

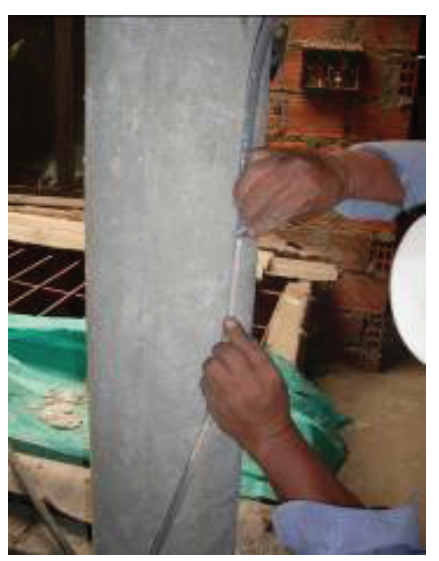

Segunda persona marcando el punto donde indica el nivel del agua, con respecto al primer sitio.

Figura 4. Trasladando medidas ("tirando nivel"), con la manguera de nivel. A3 y Ayudante. Inicio del levante de una pared divisoria. 
Referente a la estimación de longitudes menores a un metro, se hallaron sólo dos métodos similares en la gran mayoría de los albañiles entrevistados: "La cuarta", que es la longitud que existe desde el dedo meñique al dedo pulgar de la mano cuando ésta se tiene abierta en su totalidad; conocida así, porque en promedio equivale a la cuarta parte de un metro, es decir, aproximadamente, $25 \mathrm{~cm}$. Este método de medida es aplicado en longitudes cortas, por su nivel de confiabilidad para cada albañil, pues el patrón de referencia es su propia mano, indistintamente que algunos de ellos tengan manos grandes o pequeñas. Los objetos de referencia es un método que consiste en la utilización de algún tipo de herramienta que le sea provechoso para medir una longitud corta; para ello debe conocer la medida de estos elementos para realizar una buena estimación. En los casos concretos, se encuentran ciertas herramientas, como el nivel de nano y codales; sin embargo, la creatividad juega un papel esencial en esta parte, para crear o para utilizar cualquier elemento que sirva para dicho propósito.

En la identificación de patrones de estimación por parte de los albañiles, se registra que el primer método es la observación de la distancia, "al ojo". Como segunda forma, se halló que utilizan el cuerpo como una herramienta confiable para realizar algún tipo de estimación y lo hacen por medio de "pasos" o "pasos largos". Este método tiene un margen de error pequeño o poco significativo; no obstante, se debe apuntar que un elemento importante en este método es la diferencia de estaturas entre los albañiles, por ejemplo, los albañiles A2 y A3 eran personas más bajas que los demás albañiles, en especial A2, donde el margen de error fue mayor $(20 \mathrm{~cm})$. Tal vez por estas diferencias corporales, los albañiles tienen, inicialmente, un preconcepto de una longitud, a partir de una estimación, para hacer cálculos o cuentas, pero al momento de construir se mide con herramientas. Dentro de este aspecto analizado, se percibe también la utilización de lugares u objetos de referencia para realizar otro tipo de estimaciones, elemento que se puede apreciar en los aportes realizados por A1, cuando calculó el número de baldosas que se emplearía para hacer el piso del salón de una iglesia cristiana, uso una de ellas como referencia; A3, contaría el número de casas que había desde el punto en el que se encontraba y la esquina, sabiendo que el frente de cada casa en el barrio que estaba trabajando medía 4,5m; A5 y A7, compararían el espacio que les solicitan que estimen con otros lugares donde ya han laborado y conocen las medidas, por tal motivo, es claro que el uso de este tipo de sistemas (lugares u objetos) de referencias potencializa el proceso de estimación.

Al igual que en la estimación del primer tipo de longitudes, se puede observar la aproximación que obtienen los albañiles en el cálculo de medidas. Cinco de los albañiles acudieron al empleo de su cuerpo para volver a estimar longitudes, específicamente, utilizaron lo que se conoce como "la cuarta", para medir longitudes cortas o menores a un metro, considerando que el rango de la posible medida de la cuarta para los cinco albañiles que la utilizaban era el mismo, entre 20 y $25 \mathrm{~cm}$. Por medio de su cuerpo estiman, no solo visualmente, pues existen texturas y pesos; un ejemplo, recurriendo a los dedos, para distancias más pequeñas, como para colocar un clavo, cuyo punto de entierro puede ser marcado por una uña o la separación entre ellos, por el grosor del índice. Con la observación no estiman distancias sino contexturas: la de una mezcla o la lisura de una superficie. Lo particular es que el sistema escolar tiene prohibido o no usa la percepción o partes del cuerpo: el uso de los dedos cuando se pregunta por las tablas de multiplicar, puede ser objeto de burla. Pero ¿cómo hace la gente para calcular cuántos meses hay desde mayo hasta noviembre?

¿Tiene alguna incidencia la altura o fisiología del albañil en la construcción? No, porque como se ha dicho previamente, una cosa es la estimación, como proceso de ubicación y de manejo previo del espacio y el plano y, otra es la medición como proceso de garantía de una certeza. Otro apunte para resaltar en este análisis es lo que hacía el ayudante de A5 (AA5) al momento de formar los castillos o las estructuras de hierro, que sirven de soportes en los cimientos, porque usaba "la cuarta" para lograr que hubiese la misma distancia entre cada uno de los flejes de "los castillos", es decir, se puede observar que se practica de forma implícita una transformación geométrica, como la traslación de medidas, aprovechando la mano. En esto cree toda la sociedad, hasta el dueño de la casa, menos la escuela.

Otro de de los aportes que se puede percatar es el ejercicio realizado por A2, al momento de estimar longitudes cortas, porque no recurre a partes de su cuerpo sino a la aplicación de un elemento externo, pues tomó un pedazo de papel de bolsa de cemento y lo superpuso sobre la cerámica hasta obtener la longitud de uno de sus lados, argumentando que así podría llevar esa medida donde él quisiera. La versatilidad al usar el papel "embaldosado" implica obtener medidas precisas y de forma rápida, pues basta con doblarlo simétricamente. Se logró advertir que una de las primeras opciones de instrumentos de medidas no graduadas eran objetos o herramientas que, a pesar de no ser tasadas, se les conoce su longitud o medida; por ejemplo, los obreros nombraban utensilios, como codales, reglas de repello, entre otros, de tal manera que éstos les permite realizar la estimación de longitudes, contornos o superficies planas.

De igual manera, en A3 se apreció un aspecto interesante cuando mencionó el uso de una cuerda, a la cual, se le transfiere la longitud que hay desde la pinza que hace el dedo pulgar con el índice, teniendo el brazo estirado hasta el 
centro del pecho; esto, según A3, mide un metro y de hecho, en Colombia, esto es una creencia popular. Esta cuerda tendría ventajas con respecto a un codal de un metro, ya que es de fácil manejo y mayor movilidad, porque se obtiene $50 \mathrm{~cm}$ muy fácilmente al doblar la cuerda por la mitad y así sucesivamente. Aquí se encuentra un proceso diferente, pues el cuerpo sirve como referencia para transferir una medida no graduada a un objeto.

Uno de los elementos más significativos en esta investigación es la construcción implícita, por partes de los albañiles, de las nociones de medir y de estimar, que se evidenció no sólo por las aclaraciones y las afirmaciones de ellos al momento de realizar ciertas estimaciones o medidas, sino también en el desarrollo de algunas actividades de su oficio. Relativo a las noción de estimar, los albañiles usaban expresiones, entre muchas otras, como "le falta un poquito de...", "no está bien", "no es exacto", "más o menos", "casi", "aproximadamente", "es un promedio", "ahí quedó bien", "ahî", "listo", "ya". Relativas al concepto de medir expresaron: "medir es comparar una cosa con otra", "usar el metro", "pues es saber cuánto hay, por ejemplo, de allí a allá" y ċcómo sabe cuánto hay?, "Pues... uso el metro" y ċcómo lo hace con la arena para una mezcla?, "uso la pala y uno ya sabe como es la cosa, o...".

Con base en los análisis de los elementos determinados y lo expuesto por D'Ambrosio, en la entrevista señalada con anterioridad, cuando asegura que uno de los grandes objetivos de la etnomatemática es llevar esas prácticas a la escuela y contribuir a una enseñanza mejor, se puede deducir que los aspectos que se deben considerar para la elaboración de una situación problema en búsqueda de la construcción de la noción de estimación por parte de los estudiantes de grados sexto y séptimo de la educación secundaria son los siguientes: retomar lo mencionado por el profesor Ubiratan D'Ambrosio donde sustenta que el trabajo de la Etnomatemática no es pasar al alumno las teorías matemáticas existentes, que están congeladas en los libros para que él las repita, no. Debe ser una práctica, una cosa viva, hacer matemáticas dentro de las necesidades ambientales, sociales, culturales y dar un espacio para la imaginación, para la creatividad. Entonces, se utiliza literatura, juegos, cinema, todo eso, para ver en ellos componentes matemáticos, en otras palabras y a juicio de los autores de este artículo, se puede decir que las actividades que se presenten en la situación problema deben ir más allá del aula de clase, es decir, crear un buen ambiente de aprendizaje, donde los estudiantes sean motivados para la construcción del objeto que se desea movilizar, también teniendo en cuenta lo expuesto por Obando $\mathcal{E}$ Múnera (2003), que dicen que una situación problema la podemos interpretar como un contexto de participación colectiva para el aprendizaje, en el que los estudiantes, al interactuar entre ellos mismos, y con el profesor, a través del objeto de conocimiento, dinamizan su actividad matemática, generando procesos conducentes a la construcción de nuevos conocimientos. Todo esto conlleva a plantear algunas preguntas en torno a la educación: ¿’Por qué los estudiantes no tienen la posibilidad de crear instrumentos de medición diferentes a los tradicionales que se presentan en las clases de geometría?, ¿̇por qué en las clases de geometría o matemáticas los estudiantes no pueden usar partes de su cuerpo para medir o hacer estimaciones, cuando en la cotidianidad sí lo hacen?, acaso ¿no es posible establecer equivalencias o relaciones con patrones de medidas escolares y aquellos que se emplean en el saber popular?, ¿no es posible hacer una clase de geometría donde se involucre la medición y la estimación solo con el lenguaje verbal, es decir, que se privilegie la matemática oral, como sucede en la calle?

La etnomatemática de los albañiles no aporta solamente elementos de reflexión para la construcción de la noción de estimación y de medición sino, también, para otros objetos matemáticos, en los cuales, se puede desarrollar los otros tipos de pensamiento matemáticos. Lo que aquí se ha ilustrado es apenas una muestra de la complejidad de la lógica de construcción que los albañiles emplean en ese entramado de gestos, de expresiones a medias y de la utilización de herramientas comerciales y artesanales. Al tener en cuenta el tema de los albañiles, valdría la pena analizar la siguiente pregunta: ¿Cómo se respondería a la pregunta, para qué sirven las matemáticas? Por lo general, se responde que ayudan a pensar, a razonar, a mejorar la abstracción, a organizar un discurso de manera lógica. Esto es correcto y más cuando leemos a Duval (1999). Pero no es suficiente.

A partir de los aportes de las etnomatemáticas, se puede agregar, a esta respuesta, que las matemáticas permiten el reconocimiento del otro por medio de su forma de interpretar cosmológicamente su mundo, que las matemáticas son limitadas porque tienen su propio contexto, hace que grupos sociales o culturales o prácticas sociales creen sus propios lenguajes, símbolos, expresiones gestuales y hace que todos los seres humanos seamos iguales, porque somos capaces de desarrollarla. Las matemáticas, se crean porque el medio socio-cultural de un individuo así lo exige. En este sentido, uno de los aspectos más importantes es determinar cuáles son los significados que construyen los estudiantes en la clase y la relación que los conceptos escolares tienen con el entorno socio-cultural de éste.

Se puede decir, entonces, que la Educación Matemática colombiana sigue a espaldas de su entorno socio-cultural. Los ejemplos y "significados" de situaciones repetitivas, se 
marcan a cada momento y se cree que el mejor estudiante de la clase es aquel que sabe aplicar a la perfección las leyes, las propiedades o los teoremas. Es muy fácil escuchar en problemas de aplicación "una partícula que se mueve...", pero ćqué entenderá el joven con la palabra partícula? Tampoco se trata de llevar a las aulas de clase la sub-realidad de las cosas, es decir, si estamos en el departamento de Boyacá, no se trata de colocar ejemplos con papas o, en el Valle del Cauca, con la caña de azúcar o, en Pasto, con el cuy, se trata de llevar, junto con la magnitud del ejemplo, su raíz, su significado social y cultural, su historia y sus valores. Es eso a lo que apunta el Programa de etnomatemática, una educación matemática más arraigada con la cotidianidad, explicada desde allá, partiendo de la lógica de construcción que genera cada situación, poniéndose así más al alcance de las niñas y niños, para que ellos puedan acercarse mucho a las matemáticas escolares, que solo son escritas. Esto es apenas el comienzo de una mejor y más profunda discusión sobre las relaciones de esas matemáticas externas, que algunos llaman no didácticas o no académicas, con la educación matemática escolar.

Conflicto de intereses: Los nombres de los albañiles fueron cambiados para proteger su identidad. Financiación: La financiación de esta investigación corrió por cuenta de los investigadores. Se considera que ella pudo llegar a $\$ 100.000$, por concepto de transporte.

\section{BIBLIOGRAFÍA}

1. CHAMORRO, M.; BELMONTE, J. 1994. El problema de la medida: Didáctica de las magnitudes lineales. Edi. Síntesis S.A. Serie: Matemáticas: Cultura y Aprendizaje. No 17. (Madrid). 152p.

2. BISHOP, A. 1999. Actividades relaciones con el entorno, y cultura matemática. En: Enculturación matemática, la educación matemática desde una perspectiva cultural. Edi. Paidós Ibérica S.A. p.39-84

3. BISHOP, A. 2005. Aproximación sociocultural a la educación matemática. Ed. Merlín I.D. Universidad del Valle. Instituto de Educación y pedagogía. (Cali). 199p.

4. BLANCO, H. 2008. Entrevista al profesor Ubiratan D’Ambrosio. Rev. Latinoamericana de Etnomatemáticas. 1(1):21-25.

5. DELFINO DA SILVA, M.A. 2007. A Etnomatemática em uma sala da eja: a experiência do pedreiro. Disponible desde Internet en: http://www.pucsp.br/pos/edmat/ $\mathrm{mp} /$ SILVA_maria_aparecida_delfino.html (con acceso el 14/03/11).

6. DUVAL, R. 1999. Semiosis y pensamiento humano. Registros semióticos y aprendizajes intelectuales. Traducción: Myriam Vega Restrepo. Origen: (C) Peter Lang S.A. Universidad del Valle. Instituto de Educación y Pedagogía. Colombia. 310p.

7. FRANÇOIS, K.;KERKHOVE, B.2010. Ethnomathematics and the philosophy of mathematics (education). En: Löwe, B.; Müller, T. (eds.) PhiMSAMP. Philosophy of Mathematics: Sociological Aspects and Mathematical Practice. College Publications. (London). Texts in Philosophy. 11. p.121-154.

8. GLAVAM, C. 2003. Etnomatemática, currículo e práticas sociais do "mundo da construção civil". Disponible desde Internet en: http://cimm.ucr.ac.cr/ciaem/ memorias/xi_ciaem/223_etnomatematica.pdf (con acceso el 20/03/11).

9 GOETZ, J.; LeCOMPTE, M. 1998. Etnografía y Diseño Cualitativo en Investigación Educativa. Ed. Morata. (Madrid). 280p.

10. KNIJNIK, G. 2006. Educacao matemática, culturas e conhecimento naluta pela terra. EDUNISC. (Santa Cruz do Sul). 239p.

11. LUNKES, A. 2004. Etnomatemáticas: sobre a pluralidade nas significacoes do programa etnomatemática. Em: Machado, J.; Santos, M. Ferreira, R. eds. Etnomatemática: Papel, valor e significado. $1^{\text {a }}$ edición. Zouk (San Pablo). p.75-87.

12. MACHADO, J.; SANTOS, M.; FERREIRA, R. 2004. Etnomatemática: Papel, valor e significado. Zouk. (San Pablo). 287p.

13. Ministerio de Educación Nacional (MEN). 1998. Serie lineamientos curriculares. Disponible desde Internet en: http://www.mineducacion.gov.co (con acceso el 10/03/10)

14. OBANDO, G.; MÚNERA, J. 2003. Las situaciones problema como estrategia para la conceptualización matemática. Rev. Educación y Pedagogía. (Medellín). 15(35):183-199.

15. PARDAL, E. 2008. Um estudo de etnomatemática: a matemática praticada pelos pedreiros. Disponible 
desde Internet en: http://repositorioaberto. univab.pt/bitstream/10400.2/1359/1/U.A.A\%20 Matem\%C3\%A1tica\%20Praticada\%20pelos\%20 PedreirosEug\%C3\%A9nia\%20Pardal.pdf (con acceso el 14/03/11).

16. POSADA, F. 2007. Pensamiento Métrico y sistemas de medidas: Módulo 3. Secretaría de Educación para la Cultura de Antioquia, Universidad de Antioquia. (Medellín). 129p.

17. SKOVSMOSE, O. 2004. Foreground dos educandos e a política de obstáculos para aprendizagem. En: Machado, J.; Santos, M.; Ferreira, R. eds.
Etnomatemática: Papel, valor e significado. Zouk. (San Pablo). p.103-122.

18. TRUZZI, A. 2006. Estudo da Utilização de Medidas Não-Oficiais em uma Comunidade de Vocação Rural. Disponible desde Internet en: http:// www.athena.biblioteca.unesp.br/exlibris/bd/ brc/33004137031P7/2006/mauso_apt_me_rcla.pdf (con acceso el 14/03/11).

Recibido: Septiembre 21 de 2010

Aceptado: Marzo 30 de 2011 